\title{
The Production of Sporidesmin and Sporidesmolides by Wild Isolates of Pithomyces chartarum in Surface and in Submerged Culture
}

\author{
BY JOAN M. DINGLEY \\ Plant Diseases Division, Department of Scientific and Industrial Research, \\ Auckland, Nerw Zealand \\ J. DONE AND A. TAYLOR \\ Ruakura Animal Research Station, Hamilton, New Zealand \\ AND D. W. RUSSELL \\ Plant Chemistry Division, Department of Scientific and Industrial Research, \\ Palmerston North, Nerw Zealand \\ (Received 5 December 1961)

\section{SUMMARY} \\ Four wild and two laboratory isolates of Pithomyces chartarum were \\ grown under identical conditions, in submerged and in surface culture; \\ yields of organisms and utilization of medium constituents are reported. \\ Sporidesmin was produced in submerged culture and in surface culture \\ by all the isolates examined but these showed differences of up to at least \\ 100-fold in ability to produce the metabolite. Sporidesmolides were not \\ isolated from cultures which did not sporulate.
}

\section{INTRODUCTION}

The production of sporidesmin and sporidesmolides by Pithomyces chartarum in surface culture was studied by Done, Mortimer, Taylor \& Russell (1961), who showed that sporidesmin appeared in the cultures on the third day of incubation. They were unable to show significant differences in the amount of the metabolite present in the cultures for 4 days thereafter. By contrast the production of sporidesmolides was proportional to the growth of the organism and particularly to the number of conidia produced. It has been postulated that $\boldsymbol{P}$. chartarum is implicated in the aetiology of facial eczema and in this connexion Russell, Synge, Taylor \& White (1962) showed that the depsipeptides (Shemyakin, 1960) isolated from pasture where the disease occurred were identical with the sporidesmolides. These results were obtained by using an organism (strain $\mathrm{C}$ ) isolated as a distinctly highsporing laboratory sector from a culture obtained from a pasture at Claudelands, Hamilton, New Zealand in March 1958 (Thornton \& Ross, 1959). It was therefore of interest to determine whether sporidesmin and sporidesmolides were produced by field isolates and also whether such isolates differed in ability to produce these metabolites. It is known (Morton, 1960) that many fungi produce fewer spores in submerged culture than in surface culture. Thus it is possible to compare the same organism growing on the same medium at the same temperature under conditions 
where one culture produces many times the number of conidia produced by the other. These contrasted experimental conditions were used with $P$. chartarum in an attempt to obtain further information on the possible relationships of sporidesmin- and sporidesmolide-production with sporulation.

\section{METHODS}

Organisms. Pithomyces chartarum occurs as a common saprophyte on debris in pastures in the north of New Zealand. Many isolates were obtained from pasture debris and from agar plates exposed in pastures where stock were prone to facial eczema. From these field isolates four (S73a, SA 10b, SA 19a and SA 26a(i)) were selected as single spores for detailed study. In addition, two isolates (C, SA 26a(ii)) were obtained as single spores from high-sporing sectors of laboratory cultures of the fungus. The isolates differed from one another in small morphological and cultural characters. The following descriptions are based on 21-day cultures on potato glucose agar incubated at $24^{\circ}$. Isolate $\mathrm{C}$ (= strain $\mathrm{C}$, Done et al. 1961; Commonwealth Mycological Institute, Kew, England, herbarium no. 74473) was obtained from Dr R. H. Thornton, Soil Bureau, Department of Scientific and Industrial Research, Wellington, New Zealand. Ross (1960) stated that isolate C is a high-sporing isolate which rarely sectors in culture with optimum growth at about $27^{\circ}$. Cultures of isolate $\mathrm{C}$ were flat, zonate, dark coloured when over 10 days old with some floccose superficial mycelium, pigmented verrucose mycelium; intercalory chlamydospores were common among the mycelium. Conidia were formed scattered in large numbers among the superficial mycelium; they were typically muriform, with 3 transverse septa (rarely 2 ) and with 2 or more longitudinal septa; these conidia were $17-25 \times 9 \cdot 5-13 \mu$ in size. Isolate $S 73 a$ was isolated from a sporing colony on debris of Paspalum dilatatum (May 1959, Mount Albert, Auckland). Like isolate $\mathrm{C}$ optimum growth was obtained between $25-28^{\circ}$ but the cultures were light coloured with more superficial floccose mycelium. Dark chlamydospores and dark verrucose mycelium were commonly produced. Conidia were formed in small clusters in among the floccose superficial mycelium; conidia (21-29 $\times 9 \cdot 5-13 \mu)$ were muriform with 2 but more usually 3 , transverse septa. Isolates SA10b, SA19a and SA26a(i) were collected from agar plates exposed at Mahuta, Dargaville, New Zealand, in late January and February 1960; unlike isolates C and S73a they were unstable in culture and sectors were common. In sporing cultures conidia were formed superficially but scattered and in densely compacted clusters. Isolate SA 10b rarely produced conidia during incubation for 14 days. Cultures were almost colourless as dark mycelium and chlamydospores were rarely formed. Maximum growth was observed at 20-28 ${ }^{\circ}$. Cultures of isolate SA 19a were moderately sporing, floccose zonate; conidia were irregular in size varying from 16 to $35 \mu$ in length and 9.5 to $13 \mu$ in width, and with 2-5 (commonly 3 ) transverse septa. Its growth was limited at $26^{\circ}$. Sectoring was common in cultures of isolate SA 26a(i) which grew equally well at $28^{\circ}$ as at $24^{\circ}$. Conidia were regular in size varying from 19 to $27 \mu$ long and 9.5 to $13 \mu$ wide, with 3 transverse septa. Like isolate $C$, isolate SA 26a(ii) was selected from a distinct high sporing sector on a culture of isolate SA 26a(i) on potato-glucose agar. Isolate SA 26a(ii), unlike its parent, produced conidia that were erratic in size $(16-30 \times 7-14.5 \mu)$ but like its parent, it commonly showed sectoring and it grew equally well at $28^{\circ}$ as at $24^{\circ}$. 
Cultivation of organisms. Isolates were grown on potato glucose agar which was prepared by covering potatoes (chopped, $200 \mathrm{~g}$.) with water $(300 \mathrm{ml}$.). The mixture was boiled for 30 min., strained through muslin, then glucose $(20$ g.), agar (Davis 'Bacteriological', Christchurch, New Zealand; 12 g.) and Marmite (0.2 g.) added. The solution ( $\mathrm{pH} \mathrm{5 \cdot 5-5 \cdot 8)}$ was diluted to 11 . with water. The isolates S 73a, SA 19a, SA26a(i) and SA26a(ii) were inoculated on to potato glucose agar films on the inside of $15 \times 1 \mathrm{~cm}$. test tubes. The cultures were incubated 21 days at $25^{\circ}$, were freeze-dried and then sealed. Freeze-dried cultures were stored at $0^{\circ}$. Isolate $\mathbf{S A} 10 \mathrm{~b}$ was inoculated on to potato glucose agar slopes that were incubated for 21 days at $25^{\circ}$, then sealed and stored at $-40^{\circ}$. A sample of each culture thus prepared was transferred aseptically to potato carrot agar (Done et al. 1961). Plates were incubated for 14 days at $25^{\circ}$, treated with sterile $0.05 \%(v / v)$ Lissapol $\mathbf{N}$ (Imperial Chemical Industries Ltd., 16, The Terrace, Wellington, New Zealand; 5 ml.), rubbed with a stout platinum loop and the suspension obtained transferred aseptically to a $25 \mathrm{ml}$. blood bottle. The suspension was diluted with $0.05 \%(\mathrm{v} / \mathrm{v})$ Lissapol $N$ to a final concentration of $6 \cdot 10^{5}$ spores $/ \mathrm{ml}$. except in the case of isolate SA $10 \mathrm{~b}$ where no spores were seen in the inoculum. The inocula $(0 \cdot 1 \mathrm{ml}$.) were added aseptically to the culture vessels. Inocula of isolate $\mathrm{C}$ were prepared as described previously.

Surface cultures on potato carrot medium. Thirty-six l. of potato carrot medium (Done et al. 1961; containing, g./l.: potato carrot solids, 20-25, sugars 10, nitrogen $0.5 ; \mathrm{pH} 5.0$ ) were prepared and $150 \mathrm{ml}$. added to each of 240 pint milk bottles. Forty milk bottles were used for each isolate. After inoculation the 240 culture vessels were stacked on a plane slightly inclined from horizontal. The vessels of each isolate were distributed at random throughout the stack which was incubated at $25^{\circ} \pm 1^{\circ}$ for 7 days.

Submerged cultures ( $i$ ) comparative experiments. Potato carrot medium (7-2 l.) was prepared and distributed in $150 \mathrm{ml}$. amounts into each of 48 Erlenmeyer flasks (capacity $750 \mathrm{ml}$.); 8 flasks were used for the growth of each isolate. The 48 flasks were distributed at random on the shaker after inoculation. They were shaken in an horizontal plane at $110 \mathrm{rev} . / \mathrm{min}$., each flask describing a circle of radius $5 \mathrm{in}$. Cultures were incubated at $25^{\circ} \pm 1^{\circ}$ for $4-7$ days.

Submerged cultures (ii) sporidesmin production experiments. Forty-nine Erlenmeyer flasks (capacity $750 \mathrm{ml}$.) each containing potato carrot medium (150 ml.) were inoculated with isolate $\mathrm{C}$ or isolate $\mathrm{S} 73 \mathrm{a}$ and the cultures were grown for 4 days under the conditions described in the previous paragraph for comparative experiments.

\section{Harvesting and extraction}

Surface cultures. Six vessels from each group of 40 were selected at random; 2 of these were used as inoculum for potato glucose plates. These plates were incubated for 21 days at $24^{\circ}$ and then examined for contaminants. Cultures from 4 vessels were filtered and sugar (Hanes, 1929), nitrogen (Keldjahl), pH value and dry-weight determinations made on the separate filtrates. The residual fungus was washed with water, dried in a vacuum oven at $20 \% .5 \mathrm{~mm} . \mathrm{Hg}$ pressure and weighed. The dry fungal tissue was ground to a powder, thoroughly mixed and spore numbers and sporidesmolide estimations done on weighed samples. The remaining 34 vessels of each isolate were combined and the resulting 6 batches of culture extracted con- 
currently in similar equipment. The extraction and estimation of the sporidesmin content of the extracts obtained were carried out as described by Done $e t$ al. (1961).

Submerged cultures. In the comparative experiments 2 vessels of each group of 8 were used as inocula for potato glucose agar plates. Plates were incubated and examined for contaminants as described above. The contents of the remaining 6 vessels of each group were bulked and filtered. Nitrogen, sugar, $\mathrm{pH}$ value and dry-weight determinations were made on the filtrates. The residual mycelium was washed with water, dried in a vacuum oven at $20^{\circ} / 0.5 \mathrm{~mm}$. $\mathrm{Hg}$ pressure and weighed. Weighed samples of the dry powdered mycelium were used for estimations of sporidesmolides and spore numbers. In sporidesmin production experiments 4 of the 49 vessels were selected at random and used for estimations of medium constituents, amount of organism and for checking the homogeneity of the isolate. The remaining 45 cultures were combined, extracted and the sporidesmin content of the extract determined iodometrically and by the tissue-culture toxicity method (Done et al. 1961).

Sporidesmolides were estimated by a modification (suggested to us by M. E. Carruthers) of the method of Done et al. (1961) since it was found that the estimation was expedited by centrifugal separation of the material insoluble in diethyl ether. Chloroform extracts of the residue after centrifugation were satisfactorily filtered by gravity through charcoal. The amino acid composition of sporidesmolides from the different isolates was determined by the method of Russell et al. (1962).

\section{RESULTS}

The utilization of medium constituents and yields of fungus, spores, sporidesmin and sporidesmolides in a typical set of comparative surface cultures are summarized in Table 1. Five comparative experiments of this sort were carried out; the results obtained in each set were similar. The yields of sporidesmin given in Table 1 were obtained by iodometric assay and tissue-culture toxicity tests of extracts of cultures prepared under similar conditions. Good agreement between the two assay procedures was observed except in the case of isolate SA 10b where the figure obtained by the iodometric procedure was 5-10 times greater than that obtained by the tissue-culture toxicity test. The biological potency of an extract of 2001 . of culture of isolate SA10b was therefore determined by the corneal opacity test (kindly carried out for us by Mr P. H. Mortimer; Done et al. 1961). The result obtained agreed with that obtained by the tissue-culture toxicity method. Thus this figure is given in Table 1.

In submerged culture growth of all isolates was more rapid than in surface culture. The growth of isolate $\mathbf{C}$ during the 7 days after inoculation is shown in Fig. 1 and is typical of the other 5 isolates. After the fourth or fifth day breakdown of fungal tissue was noticed by microscopical examination, decreased weight of mycelium on harvest and an increase in the nitrogen concentration of the spent culture medium. The utilization of medium constituents and the production of spores and sporidesmolides are therefore given for 4-day cultures in Table 2. Three comparative experiments of this sort were done in submerged culture; similar results were obtained in all. In one of these half the cultures (i.e. 4 vessels of each isolate) were harvested 4 days after inoculation and the remainder at 7 days. Spores 
Table 1. Utilization of medium constituents, and yields of organism, spores, sporidesmin and sporidesmolides by six isolates of Pithomyces chartarum in surface culture for 7 days at $\mathbf{2 5}^{\circ}$

\begin{tabular}{|c|c|c|c|c|c|c|c|c|}
\hline \multirow[b]{2}{*}{ Isolate } & \multirow[b]{2}{*}{$\begin{array}{c}\text { Final } \\
\text { pHI } \\
\text { value* }\end{array}$} & \multicolumn{4}{|c|}{ mg./ml. } & \multicolumn{3}{|c|}{ mg./l. } \\
\hline & & $\begin{array}{l}\Delta \text { dry } \\
\text { wt. }\end{array}$ & $\Delta$ sugar & $\Delta \mathbf{N}$ & $\begin{array}{l}\text { Mycelium } \dagger \\
\text { dry wt. }\end{array}$ & $\begin{array}{c}\text { Spores } \\
\left(\times 10^{-5} /\right. \\
\mathrm{ml} .)\end{array}$ & $\begin{array}{l}\text { Sporides- } \\
\text { molides }\end{array}$ & $\begin{array}{l}\text { Sporides } \ddagger \\
\text { min }\end{array}$ \\
\hline C & 6.57 & $14 \cdot 0$ & 6.5 & 0.45 & 6.2 & 110 & 110 & 0.7 \\
\hline S73a & $6 \cdot 25$ & $12 \cdot 2$ & 4.5 & $\mathbf{0} \cdot \mathbf{3 1}$ & 6.7 & lost & 150 & $2 \cdot 1$ \\
\hline SA10b & $6 \cdot 1$ & $9 \cdot 4$ & $\mathbf{2 \cdot 9}$ & 0.23 & $5 \cdot 8$ & $0 \cdot 0$ & 0.0 & $0 \cdot 005$ \\
\hline SA 19a & $6 \cdot 15$ & $13 \cdot 6$ & 4.7 & $0 \cdot 4$ & 6.5 & 180 & 155 & $1 \cdot 4$ \\
\hline SA 26a (i) & $6 \cdot 33$ & $12 \cdot 6$ & $5 \cdot 2$ & $0 \cdot 39$ & $6 \cdot 6$ & 21 & 43 & 0.3 \\
\hline SA26a(ii) & $6 \cdot 12$ & $12 \cdot 0$ & $4 \cdot 8$ & $0 \cdot 33$ & $6 \cdot 0$ & 140 & 140 & $0 \cdot 6$ \\
\hline
\end{tabular}

$\Delta$ dry wt., $\Delta$ sugar and $\Delta \mathrm{N}$ refer to the decrease in dry weight, sugar and nitrogen concentration in the medium before inoculation and after harvest. * Of culture filtrate after incubation for 7 days, † Weight of fungus after harvesting and drying to constant weight. † The average of values obtained by iodometric assay and tissue-culture toxicity test except in the case of isolate $\mathrm{SA} 10 \mathrm{~b}$ where the latter value is given.

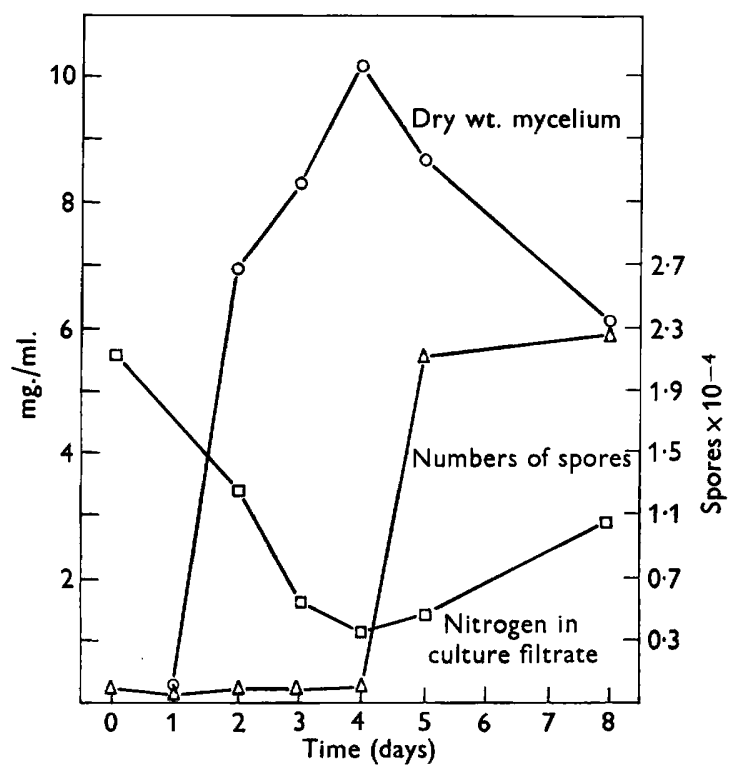

Fig. 1. Growth of Pithomyces chartarum in submerged culture. $\bigcirc-\bigcirc$, Mycelium dry weight; $\triangle-\triangle$, spore numbers; $\square-\square$, nitrogen in culture filtrate.

and sporidesmolides were present only in cultures of isolate $\mathrm{C}$ and the quantities present were the same in the 7-day-old culture as in the one 4 days old. In submerged culture isolate $\mathbf{S 7 3 a}$ often grew abnormally and formed discrete spherical colonies, or a pillar of growth in the centre of the base of the flask. The colonies

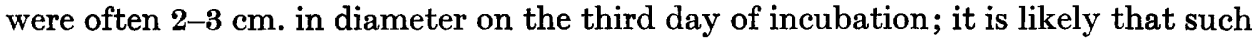
cultures were not completely 'submerged'. This would account for the appearance of conidia in cultures of isolate S73a (Morton, 1960). The occurrence of this abnormal growth was erratic and sometimes difficult to detect in vessels where very 
heavy growth had occurred. Thus the figure given in Table 2 for the number of spores produced by isolate S73a in submerged culture is open to doubt.

A strict comparison of the amounts of sporidesmin produced by different isolates in submerged culture was not possible because of the limited capacity of the shaker. The average yield of sporidesmin from 6 batches of cultures of isolate $C$ was $0.3 \mathrm{mg} . / 1$. whilst that obtained from 4 batches of isolate $S 73 a$ was $1.5 \mathrm{mg}$./l. These results were obtained on extracts of 4-day cultures since preliminary experiments indicated that cultures 7 days after inoculation contained little sporidesmin.

Table 2. Utilization of medium constituents, and yields of organism, spores and sporidesmolides by six isolates of Pithomyces chartarum in submerged culture after 4 days at $\mathbf{2 5}^{\circ}$

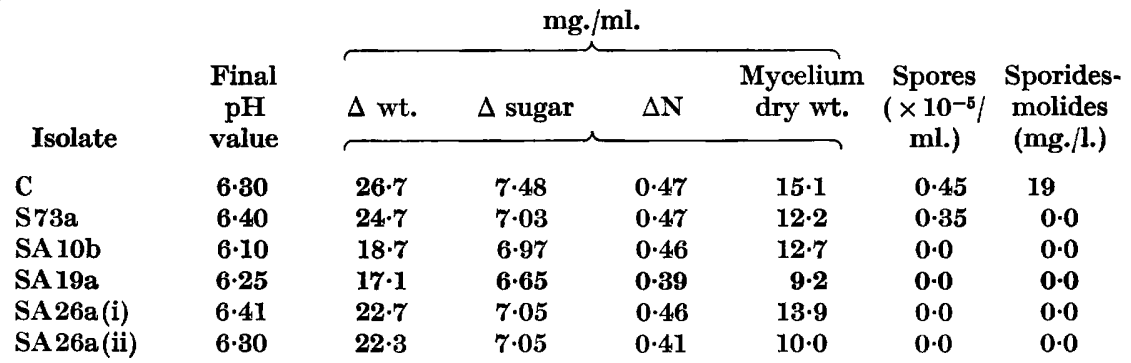

Abbreviations have the same meaning as in Table 1.

Table 3. Comparison of the weight of sporidesmolides isolated from cultures of six isolates of Pithomyces chartarum with the amount estimated polarimetrically

$\begin{array}{lccc}\text { Isolate } & \begin{array}{c}\text { Optical } \\ \text { rotation* }\end{array} & \begin{array}{c}\text { By gravimetric } \\ \text { method }\end{array} & \begin{array}{c}\text { By polarimetric } \\ \text { method }\end{array} \\ \text { C } & -205^{\circ} & 110 & 110 \\ \text { S73a } & -196^{\circ} & 158 & 153 \\ \text { SA 10b } & - & 0 \cdot 0 & 0.0 \\ \text { SA 19a } & -199^{\circ} & 138 & 134 \\ \text { SA 26a(i) } & -205^{\circ} & 28 & 28 \\ \text { SA26a(ii) } & -198^{\circ} & 173 & 167\end{array}$

* Specific optical rotations were determined in chloroform solution at $20^{\circ}$ in sodium light.

The polarimetric determination of sporidesmolides assumes constant composition of the isolated depsipeptides (Russell, 1962). Such an assumption was not necessarily tenable for cultures of different isolates. Thus the sporidesmolides from each isolate were also separated and determined gravimetrically. Some of the properties of the isolated sporidesmolides were determined. The specific optical rotations are given in Table 3. All samples sublimed in a characteristic manner when heated above $200^{\circ}$. Hydrolysates of the 6 samples contained valine, leucine, isoleucine and $N$-methyl-leucine, the relative proportions of the 4 amino acids found in each sample (in the order given) was $2: 1: 0 \cdot 2: 1$.

The effect of sequential subculture on the production of sporidesmin by isolate $\mathrm{S73a}$ in surface culture was determined. The subcultures are expressed in Fig. 2 in 
terms of $x+n$ where $x$ is a single small $(<5)$ integer and $n$ is the known number of subcultures counting from the freeze-dried culture described in the methods section. Figures were only obtained for every other subculture since between each sporidesmin production subculture the isolate was grown on potato glucose plates to make sure that contaminants were absent. Results obtained for isolate $\mathbf{C}$ grown under analogous conditions are given for comparison in Fig. 2.

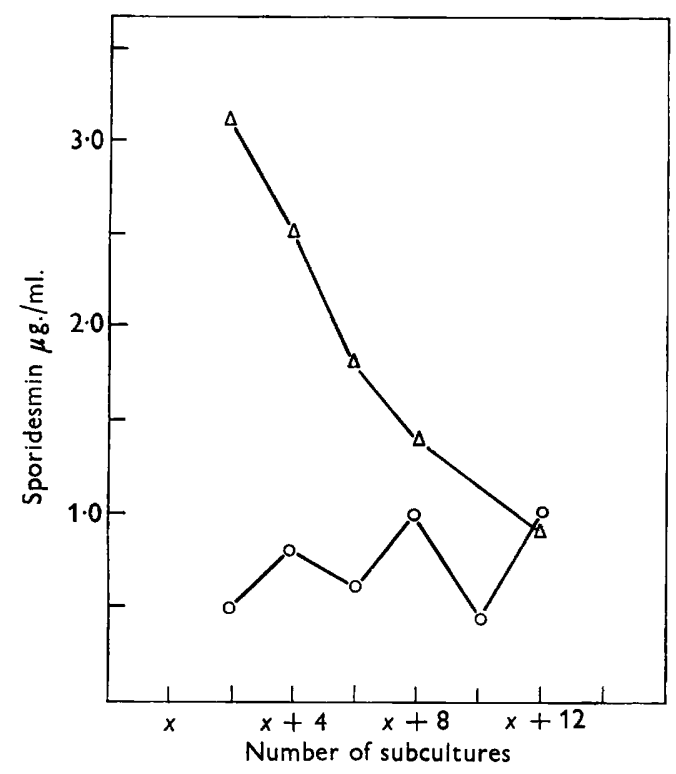

Fig. 2. Production of sporidesmin by Pithomyces chartarum isolates S73a and $\mathbf{C}$ in sequential subculture. $\triangle-\triangle$, S73a; $O-O, \mathbf{C}$; $\times$ refers to the parent freeze-dried culture as indicated in the text.

\section{DISCUSSION}

The selection of the isolates reported here was largely intuitive, taking into account geographical location, year of isolation and the differing number of conidia produced by the isolates grown on potato glucose agar. In cultures of all the isolates used in the work hyphal anastomoses were commonly seen and with four isolates (S73a, SA 19a, SA26a(i), SA26a(ii)) nuclear numbers within the cells were erratic. In addition, in cultures of the three latter isolates sectoring often occurred. Such behaviour has been observed with several species of filamentous fungi, e.g. the growth of isolates of Penicillium cyclopium on apple pulp medium (Jinks, 1952). Hence it is likely that in culture Pithomyces chartarum exhibits the 'dual phenomenum' demonstrated by Hansen (1938) for a number of Fungi Imperfecti. The results summarized in Fig. 2 are also consistent with this view; presumably they indicate the adaptive behaviour of an heterokaryon (Pontecorvo, 1946) transferred from debris of Paspalum dilatatum to sequential subculture on potato carrot medium. Two conclusions emerge. First, the physiological and morphological characters displayed by the isolates are dependent on the cultural conditions and do not justify their classification as strains, races or mutants. Thus they are referred to as isolates in this paper with a code number to indicate their origin. Secondly, in experiments 
designed to compare the growth of different isolates or their ability to produce a particular metabolite it is necessary to provide information about the number of sequential subcultures the organism has undergone in the laboratory. Thus we have subjected field isolates to a minimum number of subcultures before preparing freeze-dried parent cultures and these have been used for inocula in all comparative experiments.

The differences observed between growth in submerged and in surface culture resemble those described for other organisms (Morton, 1960). Thus in submerged cultures growth was more rapid with a shorter lag period. Conidia were produced by isolate $\mathrm{C}$ but only $\mathbf{0 . 2 5} \%$ of the number produced in surface culture were found. In submerged culture production of $1 \mathrm{mg}$. dry weight fungus required the use of about 0.04 mg. N. A similar value was observed in surface cultures of isolate SA 10b. In cultures bearing large numbers of conidia $0.06 \mathrm{mg}$. $\mathrm{N}$ was needed to produce $1 \mathrm{mg}$. dry-weight fungus. Large numbers of spores are formed in submerged and in surface culture (Done et al. 1961) only when the growth rate is decreasing; therefore sporulation in static surface culture whilst accompanied by high $\mathbf{N}$ utilization takes place only at a stage when the medium becomes depleted in this constituent. The simplest explanation of these paradoxical results seems to be that sporulation in static surface culture is stimulated by a local accumulation of metabolic products. In well mixed submerged culture breakdown of mycelium begins before such products reach a sufficiently high concentration and thus provides additional nutrient factors that can result in further mycelial growth. There is no experimental evidence for this suggestion; all aspects of the physiology of spore formation remain obscure.

Except for isolate SA10b the amount of sporidesmin recovered from surface cultures of the various isolates was of the same order. The small differences shown in Table 1 are thought to be real because they were observed in replicate experiments. From submerged cultures of isolates $\mathrm{C}$ and $\mathrm{S73a}$ sporidesmin was recovered in amounts comparable with those obtained from surface cultures of these two isolates. Thus whilst the submerged and surface cultures differed greatly with respect to the number of spores produced, sporidesmin production remained about the same.

In a previous paper (Done et al. 1961) attention was directed to the relationship between the number of spores in culture and the amount of sporidesmolides isolated therefrom. The results here given in Tables 1 and 2 amplify and confirm this relationship. No sporidesmolides were isolated from non-sporing cultures. A change from submerged culture to surface culture resulted in the formation of conidia and the isolation of sporidesmolides; but no examples were observed of the formation of one without the other with the possible exception of isolate S73a. This relationship between the numbers of spores and the amount of sporidesmolides isolated suggested that the proportion of the different depsipeptides (Russell, 1962) present might differ from one isolate to another and thus be of value in differentiating between isolates. However no differences were observed in the composition of the sporidesmolide fractions from the isolates investigated.

The authors are greatly indebted to $\mathrm{Mr}$ J. Allen whose mechanical expertise provided a shaker of exceptional reliability. Details of the design will be supplied to those interested. 


\section{REFERENCES}

Done, J., Mortimer, P. H., Taylor, A. \& Russell, D. W. (1961). The production of sporidesmin and sporidesmolides by Pithomyces chartarum. J. gen. Microbiol. 26, 207.

HaNes, C. S. (1929). An application of the method of Hagedorn and Jensen to the determination of larger quantities of reducing sugars. Biochem. J. 23, 99.

HANsen, H. N. (1938). The dual phenomenon in imperfect fungi. Mycologia, 30, 442.

JINKs, J. L. (1952). Heterokaryosis: a system of adaptation in wild fungi. Proc. roy. Soc. B, 140, 83.

Morton, A. G. (1960). The induction of sporulation in mould fungi. Proc. roy. Soc. B, 153, 548.

Ponteconvo, G. (1946). Genetic systems based on heterokaryosis. Cold Spr. Harb. Symp. quant. Biol. 11, 193.

Ross, D. J. (1960). A study of the physiology of Sporidesmium bakeri Syd.-1. Nutrition. N.Z. J. Sci. 3, 15.

Russeld, D. W. (1962). Depsipeptides of Pithomyces chartarum: the structure of sporidesmolide I. J. chem. Soc. p. 753.

Russell, D. W., Synge, R. L. M., Taylor, A. \& White, E. P. (1962). Similarity of depsipeptides from Pithomyces chartarum and from pasture samples from facial eczema areas. J. chem. Soc. p. 554.

Shemyakin, M. M. (1960). Die Chemie der Depsipeptide. Angew. Chem. 72, 342.

Thornton, R. H. \& Ross, D. J. (1959). The isolation and cultivation of some fungi from soils and pastures associated with facial eczema disease of sheep. N.Z. J. agric. Res. 2, 1002. 